\title{
Improving the Quality of Quality Measurement
}

\author{
Cheryl L. Damberg, $P h D^{7}$ and David W. Baker, MD, MPH'

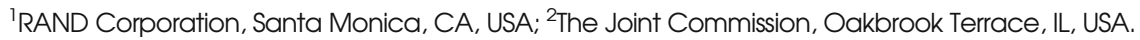

J Gen Intern Med 31(Suppl 1):S8-9

DOI: $10.1007 / \mathrm{s} 11606-015-3577-\mathrm{y}$

(c) Society of General Internal Medicine 2016

$\mathrm{T}$ he widespread, systematic use of quality measures over the past two decades has been a substantial force for change in the delivery of health care. The NCQA HEDIS measures ${ }^{1}$ and RAND Quality Assessment Tool ${ }^{2}$ set the stage for routine measurement of clinical quality. The PatientReported Outcomes Measurement Information System enabled development of patient-reported outcome (PRO) performance measures. ${ }^{3}$ The Consumer Assessment of Health Plan Survey (CAHPS) allows for systematic capture of patients' experiences of care. ${ }^{4}$ These efforts have been transformative, yet there are areas where we need to strengthen quality measurement.

The fundamental goal for quality measures should be to help providers and health systems achieve desired outcomes (i.e., prevent or slow the onset of disease, improve or slow decline in functioning, and provide care consistent with patient preferences). With this as the central goal, how can we design performance measures to do this more effectively?

\section{CREATE MORE FLEXIBLE MEASURES}

We must develop more flexible measures that recognize the complexities of patients' clinical needs and varying treatment goals. Intermediate outcome measures with fixed cutoffs to satisfy uniform numerator criteria, such as hemoglobin A1c control of $<7.0$, have proven problematic. The best treatment goal for one person may be inappropriate for another. Using an arbitrary cutoff may increase the burden of treatment for patients with minimal health gains, or lead to harm. To enable tailored measures and to provide patient-centered care, providers need to be able to capture patient preferences for treatment goals in electronic health records (EHRs). Performance could then be measured as the proportion of patients who achieve their stated goal within a specific time frame. This approach would promote shared decision-making and patientcentered care, while still holding health care teams accountable.

Greater flexibility in measurement can help prevent the unintended consequences of encouraging overuse and

Published online March 7, 2016 inappropriate care. Moving forward, EHRs should allow clinicians to easily record when a care process is not performed because the process is contraindicated or because a patient's circumstance makes her unlikely to benefit. When this is an option, physicians appear to record valid medical exceptions the vast majority of the time. ${ }^{5,6}$

Quality measurement should also respect patients' rights to refuse recommended treatments. Some believe that the ability of physicians to persuade patients to accept recommended care is an aspect of quality that would be lost if patient exceptions were allowed. This is an important concern, particularly if physicians can simply document patient refusals and not take adequate time to educate and counsel patients. Disparities could worsen as a result. However, not accounting for patient preferences abandons shared decision-making (e.g., a patient declining to take another medication) and respect for patients' health beliefs (e.g., declining influenza vaccination).

\section{EXPAND OUTCOME MEASUREMENT}

The ability of providers and other stakeholders to assess whether outcomes are improving is constrained by the limited number of outcome measures that currently exist. All clinical specialties should define the outcomes they are working to improve for acute, chronic, and palliative care, and should develop systems to measure those outcomes. Measures should include clinical outcomes, patient-reported outcomes, and patient experience of care. We need to ensure that measured outcomes are consistent with what patients deem as important. The Centers for Medicare and Medicaid Services (CMS) Hospital Inpatient Quality Reporting (HIQR) program measures 30-day mortality after stroke. However, for many patients with devastating strokes, a rapid death surrounded by loved ones is the preferred outcome rather than survival for 30 days. Failure to account for patient and family preferences in stroke outcomes creates perverse incentives that could lead to unwanted care and higher costs.

The use of patient-reported outcome measures (PROMs) as performance measures must advance. CMS's recently announced bundled payment pilot program for hip and knee replacement will require providers to report PROMs, which will be a pioneering test of the wide-scale use of PROMs for quality measurement. The biggest challenge will be the feasibility and expense of collecting outcomes data in heterogeneous practice settings with different EHRs. Web-based registries are likely to play a critical role in meeting this challenge, 
allowing the collection of data in hospitals, offices, ambulatory surgery centers, pre-operative clinics, and directly from patients through interviews by registry staff and by patients entering data over secure internet connections. ${ }^{7}$

We must also improve our ability to risk-adjust outcome measures. Using only claims data to risk-adjust fails to capture severity of illness and other important determinants of outcomes (e.g., socioeconomic status for cardiovascular disease). EHRs should be used to systematically capture patient factors needed for risk adjustment. (e.g., ejection fraction, cancer stage, socioeconomic status) with the information recorded in discrete, queriable fields. We need better methodologies for getting essential data into EHRs as well as extracting it, while at the same time not burdening physicians with data entry. Additional research is needed to develop riskadjustment methods for PROMs. For example, some providers may have more patients with depression and comorbid conditions such as substance abuse or post-traumatic stress disorder. This may make it impossible for these providers to achieve the same reduction in depressive symptoms as providers with less vulnerable patient populations.

\section{ADVANCE MEASUREMENT OF CARE PROCESSES}

Despite calls for a wholesale shift away from process measures, assessment of the delivery of recommended care should continue to play an important role. Process measures, if based on well-conducted randomized studies, identify concrete steps providers can take to improve patient outcomes, and remain useful tools for improving quality. However, we need a more responsive approach to adjust what is measured as conditions change. For example, performance on many older process measures is very high; these measures should be removed from value-based purchasing measure sets, and efforts should shift to having providers use these measures for monitoring to ensure rates remain high. As new therapies emerge with a demonstrated link to outcomes, new process measures should be rapidly developed and implemented, accompanied by aggressive quality improvement efforts to rapidly improve performance. As rates on these new measures top out, they too should be retired and used for monitoring alone.

The continued success of measuring quality will depend on our ability to do this accurately using EHRs, (i.e., electronic clinical quality measures [eCQMs]). There have been relatively few studies on the accuracy of eCQMs. Therefore, the report by Phipps ${ }^{8}$ and colleagues in this issue of JGIM offers encouraging results that such measures can be implemented and will yield accurate results. However, this study was conducted only within VA hospitals using a single EHR, and there may be differential accuracy of eCQMs across different EHRs. Absent standardization, this would make using eCQM data for comparing performance across providers in value-based payment and public reporting applications highly problematic. The studies by Saini et al ${ }^{9}$ and Farmer et al ${ }^{10}$ in this issue of JGIM also show the potential of eCQMs for monitoring overuse and patient-reported outcomes, respectively. Many more studies of eCQMs are needed, especially for more complex measures that include time stamps and radiology and laboratory data. We must find ways to make electronic quality measure work, because this offers us the prospect of inexpensive, sustainable, real-time quality measurement that can be integrated with tools in EHRs and used to drive quality improvement.

In closing, we need a dynamic system of quality measurement. To help providers continuously improve, the measurement system must also continuously improve. Moving to the next phase of quality measurement will require smarter measure construction that takes into account patient preferences and tradeoffs, better data that cuts across providers and settings and that has richer information on outcomes and patient factors affecting outcomes, and better summarization of the results of measurement in real time for use by providers to support quality improvement.

Corresponding Author: Cheryl L. Damberg, PhD; RAND Corporation, 1776 Main Street, Santa Monica, CA 90407, USA (e-mail: damberg@rand.org).

\section{REFERENCES}

1. National Committee for Quality Assurance. Healthcare Effectiveness Data and Information Set (HEDIS). 2015; Available at: http://www.ncqa.org/ HEDISQualityMeasurement/HEDISMeasures.aspx. Accessed December $10,2015$.

2. McGlynn EA, Asch SM, Adams J, et al. The quality of health care delivered to adults in the United States. N Engl J Med. 2003;348(26):26352645 .

3. National Institutes of Health. Promis $® . ~ 2015$; Available at: http://www. nihpromis.org/. Accessed December 10, 2015.

4. Agency for Healthcare Research and Quality. CAHPS Surveys and Guidance. 2015; Available at: https://cahps.ahrq.gov/surveys-guidance/ index.html. Accessed December 10, 2015.

5. Doran T, Kontopantelis E, Fullwood C, Lester H, Valderas JM, Campbell S. Exempting dissenting patients from pay for performance schemes: retrospective analysis of exception reporting in the UK Quality and Outcomes Framework. BMJ. 2012;344:e2405.

6. Persell SD, Dolan NC, Friesema EM, Thompson JA, Kaiser D, Baker DW. Frequency of inappropriate medical exceptions to quality measures. Ann Intern Med. 2010;152(4):225-231.

7. Franklin PD, Lewallen D, Bozic K, Hallstrom B, Jiranek W, Ayers DC. Implementation of patient-reported outcome measures in U.S. Total joint replacement registries: rationale, status, and plans. J Bone Joint Surg Am. 2014;96(Suppl 1):104-109.

8. Phipps M, Fahner J, Sager D, et al. Validation of Stroke Meaningful Use Measures in a National Electronic Health Record System. J Gen Intern Med. 2015. doi:10.1007/s11606-015-3562-5.

9. Saini S, Powell A, Dominitz J, et al. Developing and Testing an Electronic Measure of Screening Colonoscopy Overuse in a Large Integrated Healthcare System. J Gen Intern Med. 2015. doi:10.1007/s11606-0153569-y.

10. Farmer M, Rubenstein L, Sherbourne C, et al. Depression Quality of Care: Measuring Quality over Time using VA Electronic Medical Record Data. J Gen Intern Med. 2015. doi:10.1007/s11606-015-3563-4. 